\title{
Impact of prior sternotomy on survival and allograft function after heart transplantation: a single-center matched analysis
}

\author{
Evgenij Potapov ${ }^{1}$ and Julia Stein ${ }^{2}$ \\ ${ }^{1} \mathrm{DHZB}$ \\ ${ }^{2}$ DHZB Dienstleisungs GmbH
}

November 30, 2021

Impact of prior sternotomy on survival and allograft function after heart transplantation: a single-center matched analysis

Prior sternotomy versus primary heart transplant

Editorial

E. Potapov, J. Stein

In the presented study, the authors showed that prior sternotomy in heart transplant candidates does not impact survival after heart transplantation (reference). The analysis was performed between 106 propensity score-matched pairs (212 patients).

The results of published studies are contradictory and confusing. The small, single-center study published by Sert D, 2020, showed an increased early mortality in HTx recipients with prior sternotomy ${ }^{1}$, with no increased bleeding risk and a similar 4-year survival. In contrast, yet another single-center study with a similar design - no sternotomy vs. VAD-supported vs. prior sternotomy other than for VAD- published by Gaffey in 2015 showed no differences regarding early and long-term mortality, but a significantly higher risk of postoperative bleeding and a greater use of blood products ${ }^{2}$. A further single-center study with a comparable number of patients published in 2018 by Still S yet again showed that HTx recipients with prior sternotomy required more blood transfusions and showed an increased incidence in postoperative pneumonia, wound infection, and longer hospital stays. A stepwise multivariable regression model identified prior sternotomy as a predictor of primary graft dysfunction with a subsequently higher short-term and 1-year mortality ${ }^{3}$. However, in these studies the impact of confounding variables was not eliminated.

Having said that, the presented study attempts to overcome imbalances in confounding variables by comparing survival and complications between propensity score-matched patient groups. The matching procedure was based on established risk factors including recipients' baseline characteristics, donor age, sex mismatch, risk scores, ECLS, PVR, serum creatinine, and serum bilirubin, and ultimately produced 106 matched pairs. However, the benefit of balanced confounders is achieved at the cost of a reduced number of patients and therefore less power to detect differences between patient groups. The lack of significant differences in this study cannot merely be explained by a low power, but is instead due to the similarity of effects: Authors report a 30-day mortality of $5.7 \%$ for prior sternotomy vs. $7.5 \%$ for first-time sternotomy and no impact of prior sternotomy on long-term survival with an HR of 0.87 [95\% CI: 0.57, 1.56]. Contradicting the above-mentioned studies, these results do not even come close to indicating a trend towards worse long- and short-term survival for patients with prior sternotomy. This also holds true for long-term survival in the unmatched population of 131 patients with and 381 patients without redo surgery.

Now how about a larger registry-based analysis? An analysis based on the UNOS database comprising 
11,266 patients showed that prior sternotomy was associated with an excess $3.3 \%$ mortality and higher morbidity within the first 60 days after heart transplantation, as measured by the frequency of dialysis, drugtreated infections, and strokes. Conditional 5-year survival after 60 days is unaffected by prior sternotomy ${ }^{4}$. Five years later, another group performed an analysis of the same UNOS database (meanwhile comprising 14,730 patients) and showed again that prior sternotomy is a risk factor for worse survival after cardiac transplantation, mainly due to an increased early postoperative mortality ${ }^{5}$.

Why are we interested in knowing the effect of prior sternotomy on post-transplant outcomes? Since a prospective, randomized study is not possible due to ethical reasons, we have to rely on retrospective analyses. From a practical perspective, a comparison is meaningless - patients with prior sternotomy would never be refused for HTx due to this fact alone, even if we knew that morbidity and mortality in these patients may be higher.

In our opinion, such an analysis may be performed to identify and support the advantages of less invasive LVAD implantation regarding the outcome of later HTx, as was performed in a first analysis of 46 patients (sic!, the lowest number of patients among the discussed studies) published by Riebandt J, $2021^{6}$. The study showed that patients supported with LVAD implanted via full sternotomy required more packed red blood cells with no increased risk of bleeding, and subsequently developed more donor-specific antibodies, however, without any impact on short- and long-term survival, similar to the studies discussed above ${ }^{2,3}$.

However, the virgin chest is not comparable to that of patients supported with an LVAD implanted via a less invasive approach, even if $\mathrm{no}^{7}$ or partial sternotomy ${ }^{8}$ is performed. In either of the groups the pericardium remains intact. Regardless of the technique used, the left pleura is opened, as is the pericardium around the ascending aorta and the apex of the right and left ventricles, and the graft is placed into the pericardial space, causing adhesions making any efforts to suggest, that the HTx in the "virgin chest"is similar to that after less invasive LVAD implantation not appropriate.

Finally, the authors should nonetheless be congratulated on their outstanding surgical experience and the resulting very good outcomes in HTx - better than in the majority of centers worldwide.

References

1. Sert DE, Kervan Ü, Kocabeyoğlu SS, et al. Early and long-term results of heart transplantation with reoperative sternotomy. Turk gogus kalp damar cerrahisi dergisi 2020;28:120-6.

2. Gaffey AC, Phillips EC, Howard J, et al. Prior Sternotomy and Ventricular Assist Device Implantation Do Not Adversely Impact Survival or Allograft Function After Heart Transplantation. The Annals of thoracic surgery 2015;100:542-9.

3. Still S, Shaikh AF, Qin H, et al. Reoperative sternotomy is associated with primary graft dysfunction following heart transplantation. Interactive cardiovascular and thoracic surgery 2018;27:343-9.

4. Kansara P, Czer L, Awad M, et al. Heart transplantation with and without prior sternotomy: analysis of the United Network for Organ Sharing database. Transplantation proceedings 2014;46:249-55.

5. Axtell AL, Fiedler AG, Lewis G, et al. Reoperative sternotomy is associated with increased early mortality after cardiac transplantation. European journal of cardio-thoracic surgery : official journal of the European Association for Cardio-thoracic Surgery 2019;55:1136-43.

6. Riebandt J, Wiedemann D, Sandner S, et al. Impact of Less Invasive Left Ventricular Assist Device Implantation on Heart Transplant Outcomes. Seminars in thoracic and cardiovascular surgery 2021.

7. Potapov EV, Kukucka M, Falk V, Krabatsch T. Off-pump implantation of the HeartMate 3 left ventricular assist device through a bilateral thoracotomy approach. The Journal of thoracic and cardiovascular surgery 2017;153:104-5.

8. Nersesian G, Potapov E, Starck CT, et al. Surgical Implantation Techniques of Modern Continuous Flow Ventricular Assist Devices. Surgical technology international 2021;37:263-9. 